\title{
Resistance and aerobic exercise intervention during chemotherapy in patients with metastatic cancer: a pilot study in South Korea
}

\author{
Joo-Hwan Park ${ }^{1 \#} \wedge$, Ki Deok Park ${ }^{2 \#} \wedge$, Jin-Hong $\mathrm{Kim}^{3} \wedge$, Young Saing Kim ${ }^{1} \wedge$, Eun Young Kim ${ }^{4}$, \\ Hee Kyung Ahn ${ }^{1 \wedge}$, Inkeun Park ${ }^{1 \wedge}$, Sun Jin Sym ${ }^{1 \wedge}$ \\ ${ }^{1}$ Division of Medical Oncology, Department of Internal Medicine, Gil Medical Center, Gachon University College of Medicine, Incheon, South \\ Korea; ${ }^{2}$ Department of Rehabilitation Medicine, Gil Medical Center, Gachon University College of Medicine, Incheon, South Korea; ${ }^{3}$ Clinical \\ Rehabilitation Research Center, Korea Worker's Compensation \& Welfare Service, Incheon Hospital, Incheon, South Korea; ${ }^{4}$ Department of \\ Radiology, Gil Medical Center, Gachon University College of Medicine, Incheon, South Korea \\ Contributions: (I) Conception and design: KD Park, JH Kim, YS Kim, EY Kim; (II) Administrative support: None; (III) Provision of study materials \\ or patients: KD Park, JH Kim, YS Kim, HK Ahn, I Park, SJ Sym; (IV) Collection and assembly of data: JH Park, YS Kim, EY Kim; (V) Data analysis \\ and interpretation: JH Park, YS Kim, EY Kim; (VI) Manuscript writing: All authors; (VII) Final approval of manuscript: All authors. \\ \#These authors contributed equally to this work. \\ Correspondence to: Young Saing Kim, MD, PhD. Division of Medical Oncology, Department of Internal Medicine, Gil Medical Center, Gachon \\ University College of Medicine, 21, Namdong-daero 774beon-gil, Namdong-gu, Incheon, 21565, South Korea. Email: zoomboom@hanmail.net.
}

Background: We investigated the feasibility and safety of an exercise intervention in patients with metastatic solid cancer.

Methods: Patients scheduled to receive first-line chemotherapy for metastatic cancer with a life expectancy of $\geq 4$ months, no brain metastases, and no high risk of fracture were recruited to participate in a 12 -week, combined resistance and aerobic exercise program consisting of supervised, hospital-based $(2 \times /$ week $)$ and home-based training $(3 \times /$ week) during palliative chemotherapy. Feasibility and safety of the exercise intervention were the primary outcomes. The secondary outcomes were skeletal muscle mass and strength, functional capacity, quality of life (QoL), and fatigue.

Results: Nineteen patients were enrolled in this pilot study. Five patients withdrew consent before the exercise intervention due to fear of exacerbating cancer-related symptoms $(n=2)$, transportation issues $(\mathrm{n}=2)$, and unknown reasons $(\mathrm{n}=1)$. Ten patients $(71.4 \%)$ completed the 12 -week exercise program. Mean attendance rate of the supervised exercise sessions was $64.9 \%$ (range, 16.7-95.8\%). No adverse events or skeletal complications occurred during the supervised exercise sessions. Among participants, there were no significant changes in muscle area at the third lumbar level (mean change $=-0.7 \mathrm{~cm}^{2}, \mathrm{P}=0.869$ ) or appendicular skeletal muscle mass (mean change $=0.1 \mathrm{~kg}, \mathrm{P}=0.661$ ). The overall $\mathrm{QoL}$ assessed using the Functional Assessment of Cancer Therapy-General significantly improved post-exercise interventions $(\mathrm{P}=0.037)$. There were significant improvements in the QoL subdomains of emotional well-being and physical, social, and cognitive functions.

Conclusions: Exercise interventions are feasible and safe in patients with metastatic cancer. Exercise interventions can improve QoL and prevent skeletal muscle loss during palliative chemotherapy.

Koywords: Exercise; neoplasm metastasis; chemotherapy; muscle; quality of life (QoL)

Submitted May 30, 2021. Accepted for publication Jul 31, 2021.

doi: 10.21037/apm-21-1432

View this article at: https://dx.doi.org/10.21037/apm-21-1432

\footnotetext{
^ ORCID: Joo-Hwan Park, 0000-0002-3631-6029; Ki Deok Park, 0000-0003-1684-4737; Jin-Hong Kim, 0000-0001-9157-7902; Young Saing Kim, 0000-0003-0207-2617; Eun Young Kim, 0000-0002-2101-7982; Hee Kyung Ahn, 0000-0001-7786-7233; Inkeun Park, 00000003-3064-7895; Sun Jin Sym, 0000-0002-1340-6291.
} 


\section{Introduction}

Cancer cachexia is a multifactorial syndrome characterized by an ongoing loss of skeletal muscle mass leading to progressive functional impairment (1). Loss of skeletal muscle mass, called sarcopenia, is a major component of cancer cachexia and occurs frequently in patients with metastatic cancer (2). The multiple computed tomography (CT) images obtained during cancer treatment provide an opportunity to objectively quantify muscle loss at various time points. The presence of sarcopenia at baseline is associated with negative clinical effects, including deterioration in quality of life (QoL), increased risk of treatment-related toxicities, and reduced survival in patients with metastatic cancer (3-5). Recent studies also showed that a higher amount of muscle loss during palliative chemotherapy is associated with shorter survival $(6,7)$.

The management of sarcopenia has emerged as a key issue in cancer patients. Resistance and aerobic exercises have been shown to increase muscle strength and function and represent an attractive treatment strategy for cancerrelated sarcopenia $(8,9)$. It has been reported that building or maintaining muscle mass through exercise training is a safe and effective adjunct therapy in patients with metastatic cancer (10). However, in day-to-day clinical practice, cancer patients commonly report unmet needs with respect to information about exercise methods and access to exercise programs (11). A lot of healthcare professionals treating cancer patients have no experience with exercise regimens and fail to give specific advice to their patients. Furthermore, it is difficult to draw conclusions about the optimal duration, frequency, or intensity of exercise because the types of exercise interventions used in previous studies involving patients with metastatic cancer were heterogeneous.

To date, no prospective studies have been designed to determine the feasibility of an exercise programs for patients with metastatic cancer in South Korea. The purpose of this pilot study was to provide initial experimental data on the feasibility and safety of an exercise intervention for patients with metastatic cancer during palliative chemotherapy. We present the following article in accordance with the STROBE reporting checklist (available at https://dx.doi.org/10.21037/apm-21-1432).

\section{Methods}

\section{Participants}

Participants were eligible if they had a plan to receive firstline chemotherapy for metastatic solid cancer, were aged $\geq 20$ years, had an Eastern Cooperative Oncology Group (ECOG) performance status $\leq 2$, and a life expectancy $\geq 4$ months. Exclusion criteria were as follows: brain metastases; bone metastases with a high risk of fracture; musculoskeletal disorders that inhibit participants from exercise; symptomatic heart disease including congestive heart failure, arrhythmia, or myocardial infarction diagnosed within the last six months; and uncontrolled hypertension. The risk of pathologic fracture in patients with bone metastases was determined using Mirels' classification, which was assessed by a radiologist (12). The study was conducted in compliance with the Declaration of Helsinki (as revised in 2013) and was approved by the Institutional Review Board of Gil Medical Center (No. GBIRB2017-224). All participants provided written informed consent. This study is registered at the Clinical Research Information Service (No. KCT0003147).

\section{Exercise intervention}

The exercise intervention involved supervised and homebased exercise over 12 weeks. Supervised exercise took place in small groups of one to four participants under the supervision of an accredited physical therapist (J-H.K) twice a week in the hospital exercise room. Each session lasted approximately 60 minutes, consisting of stretching, aerobic, and resistance exercises. Aerobic exercise was performed using cycle ergometry. Its intensity was equivalent to $60-85 \%$ of each patient's maximum heart rate and that lasted 20 minutes in the first week and 30 minutes in weeks $2-12$. The age-predicted maximum heart rate was calculated by subtracting age from 220. Resistance exercise included nine types of bodyweight exercises. Resistance exercises are strength training exercises that use an individual's own weight to provide resistance against gravity. Participants were instructed to perform nine types of exercises (bridge, crunch, seated butterfly, push-up, squat, standing leg curl, shoulder press, kneeling leg extension, and bird-dog) with three sets of 10 repetitions for each exercise; for standing leg curl and bird-dog, one set was to hold the position for more than 10 seconds and repeat on the other side. The home-based exercise consisted of walking and nine resistance exercises described above and was performed three times a week. The intensity of exercise was adapted to the physical condition of the participant.

\section{Outcome measurements}

Primary outcome measures were safety and feasibility of 
the exercise intervention. Safety was assessed by recording the incidence and severity of any adverse events throughout the exercise intervention. Any adverse event occurring during a supervised exercise session was recorded by the physical therapist. Feasibility was assessed as the percentage of patients who did not complete the 12 -week exercise program (participant attrition rate) and the rate of attended exercise sessions out of the planned sessions (attendance rate). Participants wrote home-based exercise diaries that recorded the date of exercise, the number of sets performed per exercise, and adverse effects during the exercise and submitted them every week. They were also asked reasons for the non-attendance and delay of the exercise session.

Secondary outcome measures were skeletal muscle mass and strength, functional capacity, QoL, and fatigue. Secondary outcomes were assessed at baseline and postintervention. Quantitative assessments of skeletal muscle mass were performed using CT and bioelectrical impedance analysis (BIA). We quantified skeletal muscle mass $\left(\mathrm{cm}^{2}\right)$ in a single cross-sectional area at the first (L1) or third lumbar (L3) level of the lumbar spine. Skeletal muscle area was quantified using in-house software (Gachon_DeepBody developed in the Gil Medical Center, Incheon, Korea) that automatically identified skeletal muscle and calculated the muscle area on CT images (Hounsfield units: from -29 to 150 for skeletal muscle). Sarcopenia was defined as an L3 muscle index (L3 muscle area/height ${ }^{2}$ ) of $\leq 55 \mathrm{~cm}^{2} / \mathrm{m}^{2}$ for men and $\leq 39 \mathrm{~cm}^{2} / \mathrm{m}^{2}$ for women (L1 muscle index cutoffs: $46 \mathrm{~cm}^{2} / \mathrm{m}^{2}$ for men and $29 \mathrm{~cm}^{2} / \mathrm{m}^{2}$ for women) (13). Appendicular skeletal muscle mass (ASM) was assessed using direct segmental 8-point multifrequency BIA (InBody770, InBody Co., Ltd, Seoul, Korea). ASM was defined as the sum of the muscle mass in both the arms and legs.

Handgrip strength was assessed in the nondominant hand using a Jamar Plus+ Digital Hand Dynamometer (Patterson Medical, Warrenville, IL, USA). The patients performed the test while sitting comfortably with shoulder adducted and forearm neutrally rotated, elbow flexed to $90^{\circ}$, and forearm and wrist in a neutral position. For each assessment of handgrip strength, three measurements were made, and the highest score was used.

Functional capacity was measured using a 6-meter walk test (14). The testing distance included 2-meter acceleration and deceleration zones, with the inner 6-meter zone being the distance over which gait was timed. Gait speeds were calculated by dividing 6 meters by the time taken (meters/ second).

QoL and fatigue evaluation was performed using the
European Organization for Research and Treatment of Cancer Quality of Life Questionnaire Core 30 (EORTC QLQ-C30) (15), Functional Assessment Cancer TherapyGeneral (FACT-G) (16), and Functional Assessment of Chronic Illness Therapy-Fatigue Questionnaire (FACITFatigue) (17). The minimally important differences (MIDs) for interpreting the degree of change within a group were defined as follows: 10 for EORTC QLQ-C30 (18); 9 for total FACT-G (19); 10 for FACIT-Fatigue (20).

\section{Statistical analysis}

Descriptive statistics were reported as proportions or means \pm standard deviations (SDs). Differences between preand post-intervention values of continuous variables were analyzed using the paired $t$-test or Wilcoxon signed-rank test. Two-sided $\mathrm{P}$ values $<0.05$ were considered statistically significant. The analysis was performed using IBM SPSS Statistics for Windows, Version 25.0 (IBM Corp., Armonk, NY, USA).

\section{Results}

Between September 2017 and February 2018, 19 patients were enrolled in this pilot study. The baseline characteristics of the study population are presented in Table 1 . The median age was 60 years, and 10 patients $(52.6 \%)$ were men. All patients had metastatic cancer. The primary tumors were as follows: lung cancer $(\mathrm{n}=9)$, gynecologic cancer $(\mathrm{n}=5)$, soft tissue sarcoma $(\mathrm{n}=3)$, gastric cancer $(\mathrm{n}=1)$, and mesothelioma $(\mathrm{n}=1)$. The ECOG performance status was 1 in $12(63.2 \%)$ patients. Sarcopenia was present in 13 patients $(68.4 \%)$.

Five patients withdrew consent before the exercise intervention. Reasons for withdrawal include fear of exacerbating cancer-related symptoms $(\mathrm{n}=2)$, transportation issues $(n=2)$, and unknown $(n=1)$. The participant attrition rate of the 12-week exercise program was $28.6 \%$ (4/14). Four patients discontinued the exercise program due to disease progression ( $n=2$, aggravation of cancer pain; $n=1$, deterioration in performance status) and chemotherapyinduced toxicity $(n=1)$. The mean attendance rate of the supervised exercise sessions was $64.9 \%$ (range, 16.7-95.8\%). An attendance rate of $75 \%$ or higher was achieved in eight patients $(57.1 \%)$. The main reasons for non-attendance were personal reasons (27.6\%), public holidays (19.7\%), fatigue $(13.2 \%)$, and hospitalization (10.5\%). No adverse events or skeletal complications occurred during the 
Table 1 Baseline characteristics of participants ( $n=19)$

\begin{tabular}{lc}
\hline Variable & $\mathrm{n}(\%)$ \\
\hline Age (years) & $60[30-74]$ \\
Median [range] & $10(52.6)$ \\
Male & \\
BMl (kg/m $\left.{ }^{2}\right)$ & $0(0.0)$ \\
Underweight $(<18.5)$ & $8(42.1)$ \\
Normal $(\geq 18.5,<23.0)$ & $7(36.8)$ \\
Overweight $(\geq 23.0,<25.0)$ & $4(21.0)$ \\
Obese $(\geq 25.0)$ & \\
Stage $($ TNM) & $19(100.0)$ \\
IV & \\
Performance status (ECOG) & $7(36.8)$ \\
0 & $12(63.2)$ \\
1 & $0(0.0)$ \\
2 &
\end{tabular}

Cancer type

\begin{tabular}{lc} 
Non-small cell lung cancer & $8(42.1)$ \\
Small cell lung cancer & $1(5.3)$ \\
Soft tissue sarcoma & $3(15.8)$ \\
Cervical cancer & $3(15.8)$ \\
Endometrial cancer & $2(10.5)$ \\
Gastric cancer & $1(5.3)$ \\
Malignant pleural mesothelioma & $1(5.3)$ \\
First-line regimen & \\
Pemetrexed-cisplatin & $8(42.1)$ \\
Paclitaxel-platinum & $3(15.8)$ \\
Doxorubicin-cisplatin & $2(10.5)$ \\
Bevacizumab-paclitaxel-cisplatin & $1(5.3)$ \\
Etoposide-cisplatin & $1(5.3)$ \\
Capecitabine-oxaliplatin & $1(5.3)$ \\
Doxorubicin-ifosfamide & $1(5.3)$ \\
Gemcitabine-docetaxel & $1(5.3)$ \\
Pembrolizumab & $1(5.3)$ \\
Bone metastasis ${ }^{*}$ & $1(5.3)$ \\
Sarcopenia & $13(68.4)$ \\
\hline index; ECOG, Eastern Cooperative Oncology Group. & \\
\hline
\end{tabular}

supervised exercise sessions. Home-based exercise was performed at an average rate of $67.1 \%$ (24.1 sessions of planned 36 sessions).

Baseline and post-intervention values for skeletal muscle mass, gait speed, and hand grip strength are reported in Table 2. Among participants in the exercise program, there were no significant changes in the L3 muscle area (mean change, $-0.7 \mathrm{~cm}^{2}, \mathrm{P}=0.869$ ) and ASM (mean change, $0.1 \mathrm{~kg}, \mathrm{P}=0.661)$. The gait speed improved after the exercise intervention (mean change, $0.11 \mathrm{~m} / \mathrm{s}$ ) but the improvement was not statistically significant $(\mathrm{P}=0.120)$. The grip strength showed no significant change (mean change, $-2.7 \mathrm{~kg}$, $\mathrm{P}=0.075$ ).

Table 3 shows changes in patient-reported outcomes. Significant improvements were detected in the total score $(\mathrm{P}=0.037)$ and emotional well-being domain $(\mathrm{P}=0.006)$ of the FACT-G after the exercise intervention. There were also significant improvements in physical, social, and cognitive function on the EORTC QLQ-C30. However, there was no improvement in the symptom scales of the EORTC QLQ-C30 or FACIT-Fatigue scale.

\section{Discussion}

The current study is one of the first to evaluate the feasibility and safety of exercise intervention for Korean patients with metastatic cancer who underwent palliative chemotherapy. There is growing evidence that exercise interventions are safe and feasible in patients with metastatic cancer and even in the presence of bone metastases $(10,21)$. A review article reported that intervention attrition rates ranged from $6 \%$ to $58 \%$ (mean $25 \%$ ) and exercise session attendance ranged from $59 \%$ to $100 \%$ (10). The participant attrition rate and attendance rate of this study are similar to those of the previous studies. Therefore, our study showed that supervised combined aerobic and resistance exercise is feasible and safe during palliative chemotherapy.

Recent systematic reviews and meta-analyses showed that exercise interventions for patients with metastatic cancer are associated with improvement in QoL and physical function; supervised exercise interventions seem to confer more benefit than non-supervised interventions $(22,23)$. In our study, total score and emotional well-being of FACT-G improved significantly on the average more than MID. Emotional functioning of the EORTC QLQ-C30 subscales slightly increased, although not significant. Physical exercise might therefore be a suitable approach for improving QoL and reducing emotional distress in patients with metastatic 
Table 2 Skeletal muscle mass, gait speed, and hand grip strength before and after the exercise intervention $(\mathrm{n}=14)$

\begin{tabular}{|c|c|c|c|c|}
\hline Measures & Baseline, mean (SD) & Post-exercise, mean (SD) & Change, mean $(95 \% \mathrm{Cl})$ & $P$ value* \\
\hline $\begin{array}{l}\text { Appendicular skeletal muscle } \\
\text { mass }(\mathrm{kg})\end{array}$ & $18.1(4.8)$ & $18.2(4.2)$ & $0.1(-0.5$ to 0.8$)$ & 0.661 \\
\hline 6-meter walk (m/s) & $1.18(0.24)$ & $1.29(0.16)$ & $0.11(-0.03$ to 0.26$)$ & 0.120 \\
\hline
\end{tabular}

${ }^{*}$, paired $t$-test. SD, standard deviation; $\mathrm{Cl}$, confidence interval.

Table 3 Quality of life and fatigue values before and after the exercise intervention $(\mathrm{n}=12)$

\begin{tabular}{|c|c|c|c|c|c|}
\hline Measures & Baseline, mean (SD) & Post-exercise, mean (SD) & Change, mean (95\% Cl) & MID, improvement & $\mathrm{P}$ value \\
\hline Total score & $67.3(18.5)$ & $76.6(11.3)$ & $9.3(0.7$ to 17.9$)$ & 9 & 0.037 \\
\hline Physical well-being & $20.5(6.3)$ & $23.3(4.4)$ & $2.8(-1.0$ to 6.6$)$ & 3 & 0.128 \\
\hline Social/family well-being & $17.1(5.7)$ & $16.8(3.7)$ & $-0.3(-2.8$ to 2.2$)$ & 2 & 0.796 \\
\hline Functional well-being & $14.7(5.7)$ & $16.9(4.9)$ & $2.3(-1.0$ to 5.5$)$ & 3 & 0.157 \\
\hline \multicolumn{6}{|l|}{ EORTC QLQ-C30 } \\
\hline Global health status & $52.7(31.6)$ & $62.5(19.9)$ & $9.7(-7.1$ to 26.6$)$ & 10 & 0.231 \\
\hline \multicolumn{6}{|l|}{ Functional scales } \\
\hline Emotional function & $75.0(21.3)$ & $87.5(13.1)$ & $12.5(-0.2$ to 25.2$)$ & 10 & 0.053 \\
\hline Cognitive function & $80.5(24.4)$ & $94.4(10.9)$ & $13.9(2.1$ to 25.7$)$ & 10 & 0.025 \\
\hline Social function & $66.7(29.3)$ & $80.6(21.1)$ & $13.9(5.0$ to 22.7$)$ & 10 & $0.017^{\star}$ \\
\hline \multicolumn{6}{|l|}{ Symptom scales } \\
\hline Fatigue & $36.1(23.7)$ & $25.0(6.9)$ & $-11.1(-25.5$ to 3.3$)$ & -10 & 0.118 \\
\hline Nausea \& vomiting & $15.3(27.0)$ & $16.7(20.1)$ & $1.4(-18.5$ to 21.3$)$ & -10 & 0.881 \\
\hline Pain & $29.2(25.7)$ & $19.4(17.2)$ & $-9.7(-26.1$ to 6.8$)$ & -10 & 0.223 \\
\hline Dyspnea & $25.0(28.9)$ & $13.9(17.2)$ & $-11.1(-27.6$ to 5.4$)$ & -10 & $0.083^{*}$ \\
\hline FACIT-Fatigue scale & $35.5(9.4)$ & $40.7(7.6)$ & $5.2(-0.2$ to 10.5$)$ & 10 & 0.058 \\
\hline
\end{tabular}

*, Wilcoxon signed-rank test. SD, standard deviation; CI, confidence interval; MID, Minimal important difference; FACT-G, Functional Assessment of Cancer Therapy-General; EORTC QLQ-C30, The European Organization for Research and Treatment of Cancer Quality of Life Questionnaire Core 30; FACIT, Functional Assessment of Chronic Illness Therapy. 
cancer.

Fatigue is one of the most prevalent symptoms reported by patients with cancer during the course of their disease and its treatment (24). Cancer therapies, especially chemotherapy, can result in cancer-related fatigue (CRF) and exacerbate existing CRF (25). Several studies have investigated the effect of exercise on CRF as either the primary or secondary outcome, and systemic reviews and meta-analyses have documented significant improvements in CRF following exercise interventions (26). However, previous clinical studies evaluating the efficacy of exercise against CRF have mainly been conducted in patients with non-metastatic cancer or cancer survivors. The improvement of CRF in metastatic settings remains unclear. Recent systematic reviews and meta-analyses of metastatic cancer patients did not find a significant improvement in CRF (22,23). We evaluated CRF using the FACIT-Fatigue scale and EORTC QLQ-C30 fatigue subscale; there was no significant reduction in fatigue after the exercise intervention. In contrast to the curative setting, patients with metastatic cancer have an ongoing tumor burden and are influenced by ongoing chemotherapy, which might make it difficult to improve CRF. Therefore, in metastatic settings, avoiding exacerbation of CRF may be a realistic goal (22).

Muscle wasting is highly prevalent in patients with advanced cancer and is associated with an increased risk of chemotherapy-related toxicity, worse QoL, and poor prognosis $(4,5,27)$. Furthermore, administration of chemotherapy has been shown to promote depletion of skeletal muscle mass $(28,29)$; skeletal muscle loss is more frequent in patients receiving cytotoxic chemotherapy than in patients receiving molecular targeted therapy (30). Preservation of muscle mass is presented as a novel strategy to counteract chemotherapy toxicity and improve QoL in cancer patients (29). Recently, clinical studies are underway to evaluate the effect of various pharmacologic agents as well as resistance exercise on muscle mass. Based on this background, it is clinically important to accurately measure muscle mass and monitor muscle mass changes during cancer treatment. Our study evaluated changes in skeletal muscle mass using two modalities: CT scans used for tumor evaluation and BIA. In our study, there was no significant reduction in muscle mass after exercise interventions in patients receiving palliative chemotherapy.

The importance of exercise therapy in cancer patients has been suggested in many studies, but exercise therapy has not been widely implemented in a real-world setting.
A number of barriers affect the implementation of exercise interventions in patients with metastatic cancer (31). Patient-related factors include lack of awareness of the safety and benefits of exercise. In this study, patient concern was one of the main reasons for withdrawing consent before the exercise intervention. Given that patients with cancer have various cancer-related symptoms and decreased physical capabilities, exercise interventions need to be individually tailored. The implementation of an exercise program requires adequate equipment and facilities, as well as physical therapy specialists. Financial support for insurance systems, in addition to research funding, is needed for medical institutions to provide exercise programs for cancer patients.

Several limitations of our pilot study warrant consideration. The small number of participants limits statistical power to detect a significant difference. Another limitation of the study was the absence of a control group. The effectiveness of exercise on secondary outcomes could not be confirmed by comparing between patients who participated in the exercise program and those who did not. The benefits of exercise intervention in cancer patients can be defined following a randomized controlled trial with a large population. Third, thresholds for feasibility were not defined prior to the start of the study. Feasibility was determined by referring to previous studies conducted to evaluate feasibility and/or tolerance of the exercise intervention. Fourth, we did not apply an accurate figure system for indicating the intensity of the exercise like 1-RM in building our exercise program. Furthermore, adaptation of the exercise intensity was carried out, not in accordance with systemic protocol, but based on the subjective decision of physical therapist. This could be a factor that limits designing systemic exercise program. However, since each patients' strength and endurance varies, it seems rational to flexibly adopt the exercise intensity depending on the circumstance of each session. Finally, the minor adverse events were underestimated since the safety profile was not systematically recorded according to established criteria such as Common Terminology Criteria for Adverse Events (CTCAE). However, there was no serious symptom that required the termination of the exercise.

In conclusion, our results show that exercise interventions are feasible and safe in patients with metastatic solid cancer. Exercise interventions can improve QoL and prevent skeletal muscle loss and fatigue deterioration during palliative chemotherapy. Further randomized controlled studies are warranted to confirm these findings. 


\section{Acknowledgments}

Funding: This study was supported by a grant from the National R\&D Program for Cancer Control, Ministry of Health \& Welfare, Republic of Korea (Grant No. HA17C0045). The funder had no role in the study design, data collection and analysis, decision to publish, or preparation of the manuscript.

\section{Footnote}

Reporting Checklist: The authors have completed the STROBE reporting checklist. Available at https://dx.doi. org/10.21037/apm-21-1432

Data Sharing Statement: Available at https://dx.doi. org/10.21037/apm-21-1432

Conflicts of Interest: All authors have completed the ICMJE uniform disclosure form (available at https://dx.doi. org/10.21037/apm-21-1432) and report that this study was supported by a grant from the National R\&D Program for Cancer Control, Ministry of Health \& Welfare, Republic of Korea (Grant No. HA17C0045). The funder had no role in the study design, data collection and analysis, decision to publish, or preparation of the manuscript. The authors have no other conflicts of interest to declare.

Ethical Statement: The authors are accountable for all aspects of the work in ensuring that questions related to the accuracy or integrity of any part of the work are appropriately investigated and resolved. The study was conducted in compliance with the Declaration of Helsinki (as revised in 2013) and was approved by the Institutional Review Board of Gil Medical Center (No. GBIRB2017-224). All participants provided written informed consent.

Open Access Statement: This is an Open Access article distributed in accordance with the Creative Commons Attribution-NonCommercial-NoDerivs 4.0 International License (CC BY-NC-ND 4.0), which permits the noncommercial replication and distribution of the article with the strict proviso that no changes or edits are made and the original work is properly cited (including links to both the formal publication through the relevant DOI and the license). See: https://creativecommons.org/licenses/by-nc-nd/4.0/.

\section{References}

1. Fearon K, Strasser F, Anker SD, et al. Definition and classification of cancer cachexia: an international consensus. Lancet Oncol 2011;12:489-95.

2. Santilli V, Bernetti A, Mangone M, et al. Clinical definition of sarcopenia. Clin Cases Miner Bone Metab 2014;11:177-80.

3. Prado CM, Lieffers JR, McCargar LJ, et al. Prevalence and clinical implications of sarcopenic obesity in patients with solid tumours of the respiratory and gastrointestinal tracts: a population-based study. Lancet Oncol 2008;9:629-35.

4. Shachar SS, Williams GR, Muss HB, et al. Prognostic value of sarcopenia in adults with solid tumours: A meta-analysis and systematic review. Eur J Cancer 2016;57:58-67.

5. Nipp RD, Fuchs G, El-Jawahri A, et al. Sarcopenia Is Associated with Quality of Life and Depression in Patients with Advanced Cancer. Oncologist 2018;23:97-104.

6. Blauwhoff-Buskermolen S, Versteeg KS, de van der Schueren MA, et al. Loss of Muscle Mass During Chemotherapy Is Predictive for Poor Survival of Patients With Metastatic Colorectal Cancer. J Clin Oncol 2016;34:1339-44.

7. Daly LE, Ní Bhuachalla ÉB, Power DG, et al. Loss of skeletal muscle during systemic chemotherapy is prognostic of poor survival in patients with foregut cancer. J Cachexia Sarcopenia Muscle 2018;9:315-25.

8. Fuller JT, Hartland MC, Maloney LT, et al. Therapeutic effects of aerobic and resistance exercises for cancer survivors: a systematic review of meta-analyses of clinical trials. Br J Sports Med 2018;52:1311.

9. Stene GB, Helbostad JL, Balstad TR, et al. Effect of physical exercise on muscle mass and strength in cancer patients during treatment--a systematic review. Crit Rev Oncol Hematol 2013;88:573-93.

10. Heywood R, McCarthy AL, Skinner TL. Safety and feasibility of exercise interventions in patients with advanced cancer: a systematic review. Support Care Cancer 2017;25:3031-50.

11. Clifford BK, Mizrahi D, Sandler CX, et al. Barriers and facilitators of exercise experienced by cancer survivors: a mixed methods systematic review. Support Care Cancer 2018;26:685-700.

12. Mirels H. Metastatic disease in long bones: A proposed scoring system for diagnosing impending pathologic fractures. 1989. Clin Orthop Relat Res 2003;(415 Suppl):S4-13.

13. Kim EY, Kim YS, Park I, et al. Evaluation of sarcopenia 
in small-cell lung cancer patients by routine chest CT.

Support Care Cancer 2016;24:4721-6.

14. Lam HS, Lau FW, Chan GK, et al. The validity and reliability of a 6-Metre Timed Walk for the functional assessment of patients with stroke. Physiother Theory Pract 2010;26:251-5.

15. Groenvold M, Klee MC, Sprangers MA, et al. Validation of the EORTC QLQ-C30 quality of life questionnaire through combined qualitative and quantitative assessment of patient-observer agreement. J Clin Epidemiol 1997;50:441-50.

16. Cella DF, Tulsky DS, Gray G, et al. The Functional Assessment of Cancer Therapy scale: development and validation of the general measure. J Clin Oncol 1993;11:570-9.

17. Yellen SB, Cella DF, Webster K, et al. Measuring fatigue and other anemia-related symptoms with the Functional Assessment of Cancer Therapy (FACT) measurement system. J Pain Symptom Manage 1997;13:63-74.

18. Osoba D, Rodrigues G, Myles J, et al. Interpreting the significance of changes in health-related quality-of-life scores. J Clin Oncol 1998;16:139-44.

19. King MT, Cella D, Osoba D, et al. Meta-analysis provides evidence-based interpretation guidelines for the clinical significance of mean differences for the FACT-G, a cancer-specific quality of life questionnaire. Patient Relat Outcome Meas 2010;1:119-26.

20. Reddy S, Bruera E, Pace E, et al. Clinically important improvement in the intensity of fatigue in patients with advanced cancer. J Palliat Med 2007;10:1068-75.

21. Sheill G, Guinan EM, Peat N, et al. Considerations for Exercise Prescription in Patients With Bone Metastases: A Comprehensive Narrative Review. PM R 2018;10:843-64.

22. Nadler MB, Desnoyers A, Langelier DM, et al. The Effect of Exercise on Quality of Life, Fatigue, Physical Function, and Safety in Advanced Solid Tumor Cancers: A Metaanalysis of Randomized Control Trials. J Pain Symptom

Cite this article as: Park JH, Park KD, Kim JH, Kim YS, Kim EY, Ahn HK, Park I, Sym SJ. Resistance and aerobic exercise intervention during chemotherapy in patients with metastatic cancer: a pilot study in South Korea. Ann Palliat Med 2021;10(10):10236-10243. doi: 10.21037/apm-21-1432
Manage 2019;58:899-908.e7.

23. Yang J, Choi M, Choi J, et al. Supervised Physical Rehabilitation in the Treatment of Patients with Advanced Cancer: a Systematic Review and Meta-analysis. J Korean Med Sci 2020;35:e242.

24. Vogelzang NJ, Breitbart W, Cella D, et al. Patient, caregiver, and oncologist perceptions of cancer-related fatigue: results of a tripart assessment survey. The Fatigue Coalition. Semin Hematol 1997;34:4-12.

25. Karthikeyan G, Jumnani D, Prabhu R, et al. Prevalence of fatigue among cancer patients receiving various anticancer therapies and its impact on quality of life: a cross-sectional study. Indian J Palliat Care 2012;18:165-75.

26. Fabi A, Bhargava R, Fatigoni S, et al. Cancer-related fatigue: ESMO Clinical Practice Guidelines for diagnosis and treatment. Ann Oncol 2020;31:713-23.

27. Prado CM, Baracos VE, McCargar LJ, et al. Sarcopenia as a determinant of chemotherapy toxicity and time to tumor progression in metastatic breast cancer patients receiving capecitabine treatment. Clin Cancer Res 2009;15:2920-6.

28. Barreto R, Waning DL, Gao H, et al. Chemotherapyrelated cachexia is associated with mitochondrial depletion and the activation of ERK1/2 and p38 MAPKs. Oncotarget 2016;7:43442-60.

29. Pin F, Couch ME, Bonetto A. Preservation of muscle mass as a strategy to reduce the toxic effects of cancer chemotherapy on body composition. Curr Opin Support Palliat Care 2018;12:420-6.

30. Kakinuma K, Tsuruoka H, Morikawa K, et al. Differences in skeletal muscle loss caused by cytotoxic chemotherapy and molecular targeted therapy in patients with advanced non-small cell lung cancer. Thorac Cancer 2018;9:99-104.

31. IJsbrandy C, Hermens RPMG, Boerboom LWM, et al. Implementing physical activity programs for patients with cancer in current practice: patients' experienced barriers and facilitators. J Cancer Surviv 2019;13:703-12. 